\title{
LA PRÁCTICA PRE-PROFESIONAL EN LA FORMACIÓN DOCENTE INICIAL DESDE LAS POLÍTICAS DOCENTES EN PERÚ
}

\author{
LILEya ManriQue VilLaVICenCIO \\ Pontificia Universidad Católica del Perú (PUCP), Lima, Perú
}

\begin{abstract}
Resumen: En esta década, la formación docente en el Perú está atravesando cambios marcados por las políticas docentes, que apuestan por el ejercicio de una docencia en base a estándares vinculados a la Carrera Pública Magisterial. En este artículo, se ofrece un panorama de las políticas que regulan la formación de los futuros docentes para centrar su foco en la Práctica pre-profesional de los docentes que se forman en los Institutos de Educación Superior Pedagógica. Las prácticas intensivas se analizan desde su complejidad epistemológica y organizacional, en tanto espacio de aprendizaje, proceso complejo y singular, así como área del currículo que desarrolla competencias profesionales. Se finaliza con desafíos que permitan abrir el diálogo entre los agentes responsables de la formación docente en nuestro país.
\end{abstract}

Palabras-clave: Práctica pre-profesional. Formación docente inicial. Políticas docentes.

\section{SITUACIÓN ACTUAL DE LAS POLÍTICAS DOCENTES PARA LA FORMACIÓN DOCENTE INICIAL}

En las políticas docentes en el Perú se reconoce al docente como un profesional de la educación y un agente fundamental para concretar el derecho a la educación, que se evidencia en el logro de los aprendizajes y en la formación integral de los estudiantes peruanos. Asimismo, se reconoce que es crucial contar con maestros bien preparados, con competencias idóneas y ética para el ejercicio de su labor. De otro lado, el Estado asume un rol rector y regulador de la calidad del sistema de formación docente inicial y en servicio, así como de las instituciones formadoras de docentes que dependen de este. La formación docente inicial en el Perú se ubica en la educación superior y se caracteriza por un formato institucional dual, es decir, se encargan de la formación los institutos y las facultades de Educación.

La formación que se brinda en Institutos de Educación Superior Pedagógica (IESP), públicos y privados, depende del Ministerio de Educación. En el 2018, existen un total de 215 IESP a nivel nacional, $48 \%$ es público y el $52 \%$ privado, a los cuales se accede a través de una prueba escrita, como modalidad de admisión, definida por el Ministerio de Educación, para seguir estudios durante cinco años. Se egresa con el Título Profesional a nombre de la Nación en la especialidad elegida.

Asimismo, se forma docentes en las 74 facultades de Educación de las Universidades nacionales y privadas, durante diez semestres académicos o cinco años. 
Cada universidad define los procesos de admisión y el número de vacantes acorde a la Ley Universitaria $\mathrm{N}^{\circ} 30220$ y sus reglamentos internos. Se egresa con el grado de Bachiller en Educación y Licenciado en la especialidad respectiva. Existe una débil política de articulación del Ministerio de Educación con las universidades, en orden a generar acuerdos y consensos en cuanto a la visión sobre la docencia y el tipo de formación que se debe ofrecer en nuestro país.

Los IESP se rigen por un modelo curricular único, denominado Diseño Curricular Básico Nacional, aprobado en el 2010 por el Ministerio de Educación (MINEDU), mientras que las facultades de Educación disponen de autonomía para definir sus perfiles de egreso y diseño curricular de formación. Este sistema dual en la formación trae una diferenciación en las opciones laborales de los egresados. Los institutos formarían para el aula de la escuela pública, mientras que las facultades diversifican los campos laborales del futuro docente: escuela, editoriales, centros de investigación o de capacitación, organismos no gubernamentales- ONG, entre otros. Sin embargo, las prácticas pre profesionales preferentemente se concentran en las escuelas de educación básica, para ambas instituciones formadoras, por el vínculo con la titulación profesional entregada.

Un hito importante sobre la situación de la formación docente inicial en el Perú, acontece con el Censo Nacional a los Institutos de Educación Superior Pedagógica públicos, realizado en el año 2015, que permitió conocer el estado de los centros de estudios en cuanto a su personal, infraestructura y equipamiento. Ese mismo año, se levantó el Diagnóstico sobre la formación docente inicial en los Institutos de Formación Docente, que puso en evidencia que se forma bajo un currículo que enfatiza en contenidos y no en competencias; un plan de estudio atomizado ( 12 cursos por semestre de 2 horas semanales) que genera 5400 horas en 5 años; la práctica pre profesional e investigación desarticulados y se concentran al final de la formación y no secuencialmente (DIFOID, 2017a).

Ante esta situación, el Estado establece desde el año 2016, como una política prioritaria, la mejora y reestructuración de los sistemas de formación docente inicial y en servicio. Para el caso de la formación inicial, se promulgó en noviembre 2016, la Ley №30512 Ley de Institutos y Escuelas de Educación Superior y de la Carrera Pública de sus docentes y, en agosto 2017, se aprobó el Reglamento respectivo (DS Nº10-2017Minedu).

Con este marco legal, la Dirección de Formación Inicial Docente- DIFOID del Ministerio de Educación, lleva adelante la Reforma del subsistema de formación docente inicial, que implica un cambio en la gestión y el desarrollo de un nuevo currículo de formación por competencias profesionales, que mejore la calidad de la enseñanza y del servicio educativo, que ofrecen los institutos de educación superior pedagógicos, públicos y privados de todo el país (Velasco, 2018).

En la gestión, se está en tránsito de convertir a los Institutos de Educación Superior Pedagógica (IESP) en Escuelas de Educación Superior Pedagógica (EESP). Estas serán los centros especializados en la formación inicial docente para atender el servicio educativo público, que en base a un nuevo Modelo de Servicio Educativo, podrán otorgar el grado de Bachiller y el título de Licenciado, válidos para estudios de posgrado a nivel 
nacional. Por tanto, a los egresados de las nuevas EESP se les confiere un grado académico, que antes solo correspondía al egresado de universidad.

Sobre el currículo, la DIFOID (2017b) propone la construcción de un diseño curricular único para las EESP, basado en una formación por competencias, que asume un enfoque reflexivo en la formación, que incluye un componente curricular que articula formación en la práctica y la investigación. Este currículo no está concluido y se espera su implementación progresiva hasta el año 2021.

Las políticas docentes buscan el mejoramiento de la formación docente inicial, que se concreta en una nueva gestión a cargo de Escuelas de Educación Superior Pedagógica (EESP) y, de otro lado, se está en elaboración de un nuevo diseño curricular. Cabe señalar, que sigue vigente con sus potencialidades y limitaciones, el Diseño Curricular Básico Nacional para las carreras de Educación, en sus distintas especialidades del año 2010, sobre el cual ahondaremos nuestro análisis sobre el sistema construido para las prácticas pre-profesionales de los docentes que se forman en los Institutos de Educación Superior Pedagógica (IESP).

\section{LA PRÁCTICA PRE-PROFESIONAL EN LA FORMACIÓN DOCENTE INICIAL REGULADA POR EL MINISTERIO DE EDUCACIÓN}

En este apartado, se presenta principalmente, la conceptualización, finalidad y organización de la práctica pre-profesional, prevista en el currículo de la formación docente inicial vigente del 2010, así como los lineamientos y normativa que la afectan, propuesto para las instituciones formadoras públicas y privadas, que dependen del Ministerio de Educación. En cuanto a la normativa vigente que rige las Prácticas preprofesionales se analizaron las siguientes fuentes:

- Diseño Curricular Básico Nacional de la Formación Docente Inicial (año 2010)

- Lineamientos Nacionales para el desarrollo de la Práctica Pre-Profesional en carreras docentes en Institutos y Escuelas de Educación Públicos y Privados (RD0651-2010-ED)

- Guía de Práctica para Institutos y Escuelas de Educación Superior con carreras Pedagógicas (2011)

- Ley $N^{\circ} 28518$. Ley sobre Modalidades Formativas Laborales (cuando las prácticas pre-profesionales son en escuelas privadas). Reglamento aprobado.

- Decreto Legislativo $\mathrm{N}^{\circ} 140$ que Aprueba el Régimen Especial que regula las modalidades formativas de servicios en el Sector Público (cuando las prácticas pre-profesionales son en escuelas públicas). Reglamento en proceso.

Para abordar las prácticas pre-profesionales, se reconoce que la práctica en sí misma es compleja, singular y en la cual inciden múltiples factores y subjetividades. Por ello, se adapta la propuesta de Cifuentes (1999, citado por Escobar, 2007) quien distingue dos complejidades para comprender la Práctica en general: epistemológica y organizativa. En nuestro caso, la complejidad epistemológica remite a la naturaleza y finalidad de las prácticas pre-profesionales, al enfoque de la docencia y del docente en formación. La complejidad organizativa de las prácticas pre-profesionales se analiza a dos niveles: 1) la organización curricular y 2) la organización relacional o de vínculo entre diversos agentes. 


\section{COMPLEJIDAD EPISTEMOLÓGICA: ENFOQUE DE FORMACIÓN, NATURALEZA Y FINALIDAD DE LA PRÁCTICA PRE-PROFESIONAL}

La formación del docente se ubica en la racionalidad técnica, en tanto asume un enfoque por competencias, que busca que el estudiante alcance mejores niveles de dominio y logre las competencias profesionales previstas en el perfil del egresado. Asimismo, este enfoque considera como uno de sus principios curriculares: la reflexión en y desde la práctica para la reconstrucción social. Es decir, se incorpora también una racionalidad práctica:

la preparación del futuro docente como constructor de currículo, el cual se alimenta permanente de la práctica con una actitud investigativa e innovadora. Esto es, un profesional reflexivo que asume su práctica como espacio de diálogo desde la acción, más que como espacio de aplicación de teorías y técnicas, lo cual le permite cambios, ajustes, desaprender para aprender (MINISTERIO DE EDUCACIÓN, 2010a, p.22).

La Práctica es un área de formación que atraviesa todo el plan de estudios, que incluye dos sub áreas: práctica y práctica pre-profesional. La finalidad de la Práctica en el DCBN es poner "al estudiante en contacto progresivo y de creciente complejidad con la realidad educativa concreta a través del ejercicio profesional en condiciones reales, para que identifique, analice, reflexione y optimice roles, funciones y acciones inherentes al trabajo docente" (MINISTERIO DE EDUCACIÓN, 2011, p.10).

De esta manera, la práctica pre-profesional constituye el espacio propicio para que los estudiantes, futuros docentes, desplieguen las competencias profesionales que van desarrollando a lo largo de su preparación profesional, en condiciones reales. Además, se enfoca para que el estudiante "reconceptualice la teoría desde la práctica y viceversa, genere conocimiento pedagógico a través de la investigación y consolide el logro de las competencias profesionales de la carrera docente" (MINISTERIO DE EDUCACIÓN, 2011, p.10).

Se enfatiza en que la Práctica pre-profesional es una oportunidad para "aprender a ser maestro", para clarificar y consolidar la vocación profesional. Representa una síntesis de todo lo que el estudiante aprende en todas las áreas y sub áreas que intervienen en su formación, por lo que se propone la articulación del área de formación Práctica con las demás áreas del currículo. Asimismo, se espera que los estudiantes desarrollen una actitud investigadora, como una disposición para examinar críticamente su práctica, "construir sus propias teorías, validarlas en la acción y aplicar la teoría construida para mejorar su práctica" (MINISTERIO DE EDUCACIÓN, 2011, p.40), aunque esta vinculación no se expresa en la finalidad de la práctica como tal.

Los estudiantes en su práctica pre-profesional desarrollan la facilitación del aprendizaje en el aula en situación real, participan en la gestión institucional y pedagógica de la escuela; y participan en los talleres de sistematización. En lo que respecta a los talleres, estos serían los espacios en el centro de formación, para propiciar la reflexión teórica sobre la realidad encontrada, la experiencia vivida o el contraste con la teoría, con el fin de modificar sus intervenciones, sus actitudes, sus creencias, para optimizar sus 
VILLAVICENCIO, L. M.

acciones educativas como mediador, investigador-innovador en la construcción de saber pedagógico (MINISTERIO DE EDUCACIÓN, 2011, p.19).

En síntesis, desde la complejidad epistemológica, la formación docente inicial se enmarca en la racionalidad técnica y la práctica reconceptualista. Se busca formar a un docente que desde el acercamiento temprano a situaciones reales, ejercite la reflexión sistemática de su práctica y construya el saber pedagógico, desarrolle sus competencias profesionales y la identidad docente a lo largo de su carrera. En ese sentido, la práctica es considerada como: un espacio de aprendizaje donde se ejercitan las competencias profesionales; un proceso de reflexión en y sobre la práctica para cambiar esquemas mentales o el habitus profesional; un proceso que vincula la investigación-reflexiónacción e innovación para la mejora de la práctica misma.

\section{LA COMPLEJIDAD ORGANIZACIONAL DE LAS PRÁCTICAS PRE-PROFESIONALES}

Las Prácticas son reguladas considerando su organización en el diseño curricular de la formación docente inicial y su funcionamiento a través de la relación con las instituciones educativas. Por ello, esta complejidad organizativa se describe a dos niveles: 1) la organización curricular del área de la Práctica, en cuanto a su estructura, ubicación de la práctica pre-profesional como sub-área, agentes y contenidos en el currículo; 2) la organización relacional, es decir, el vínculo de la institución formadora con las instituciones que reciben a los docentes en formación para sus prácticas preprofesionales.

\section{ORGANIZACIÓN CURRICULAR DEL ÁREA DE PRÁCTICA}

En el Diseño Curricular Básico Nacional-DCBN (2010) para cualquiera de las especialidades de la educación básica, la Práctica se incorpora como un área de la formación, bajo un modelo curricular concurrente, según lo define Vaillant (2018). Este modelo curricular, a diferencia del consecutivo', los contenidos de la formación incluyendo las prácticas de enseñanza forman una única estructura curricular (Vaillant, 2018); es decir, los contenidos pedagógicos y los disciplinares se aprenden al mismo tiempo de forma integrada; las prácticas se distribuyen a lo largo de la formación con diferentes contenidos e intensidad, articuladas con la formación pedagógica y la formación especializada.

El Plan de estudios del DCBN en Perú, se estructura en dos grandes etapas: formación general (primer al cuarto semestre) y formación especializada (quinto al décimo semestre). En este plan se reconoce un área de formación denominada Práctica, que se subdivide en dos sub-áreas: práctica y práctica pre-profesional.

En la formación general, la sub área práctica se concreta en cuatro cursos: Práctica I, Práctica II, Práctica III y Práctica IV. Corresponde a una primera etapa de contacto e inicio de una observación sistemática, de diversas realidades socio-educativas de su medio y de confrontación teórica, a través de la conducción de actividades lúdico-recreativas, de proyección social, entre otras. Además, busca afianzar la vocación de servicio, liderazgo y la sensibilidad social ante la realidad educativa.

Durante la formación especializada, se denomina sub área práctica preprofesional que incluye dos etapas: "de profundización y sistematización" que comprende 
la Práctica Pre-profesional I- II, III y IV; luego culmina con la tercera tapa de "Práctica intensiva", que comprende los cursos denominados Práctica Pre profesional V y Vl, en el noveno y décimo ciclo. El conjunto de cursos de práctica pre-profesional favorecen en el estudiante su pensamiento creativo, reflexivo y crítico; promueven un ejercicio intensivo de la docencia en un aula de educación básica, según su especialidad.

Tabla 1. Organización curricular del Área Práctica en la formación docente inicial

\begin{tabular}{|c|c|c|c|c|c|c|c|c|c|c|}
\hline $\begin{array}{l}\text { Etapas } \\
\text { Formaci } \\
\text { ón }\end{array}$ & \multicolumn{4}{|c|}{ Formación General } & \multicolumn{6}{|c|}{ Formación Especializada } \\
\hline $\begin{array}{l}\text { Sub- } \\
\text { áreas } \\
\text { Práctica }\end{array}$ & \multicolumn{4}{|c|}{ Sub área práctica } & \multicolumn{6}{|c|}{ Sub área práctica pre-profesional } \\
\hline $\begin{array}{l}\text { Etapas } \\
\text { de } \\
\text { Práctica }\end{array}$ & \multicolumn{4}{|c|}{$\begin{array}{l}\text { De contacto con la realidad } \\
\text { educativa e inicio de } \\
\text { sistematización }\end{array}$} & \multicolumn{4}{|c|}{ 2. De profundización y sistematización } & \multicolumn{2}{|c|}{ 3. Práctica intensiva } \\
\hline Ciclos & I & II & III & IV & $\mathrm{V}$ & $\mathrm{VI}$ & VII & VIIII & IX & $X$ \\
\hline Curso & $\begin{array}{l}\text { Prácti } \\
\text { ca I }\end{array}$ & $\begin{array}{l}\text { Prácti } \\
\text { ca II }\end{array}$ & $\begin{array}{l}\text { Prácti } \\
\text { ca III }\end{array}$ & $\begin{array}{l}\text { Prácti } \\
\text { ca IV }\end{array}$ & $\begin{array}{l}\text { Práctica } \\
\text { Pre } \\
\text { profesio } \\
\text { nal I }\end{array}$ & $\begin{array}{l}\text { Práctica } \\
\text { Pre } \\
\text { profesio } \\
\text { nal II }\end{array}$ & $\begin{array}{l}\text { Práctica } \\
\text { Pre } \\
\text { profesio } \\
\text { nal III }\end{array}$ & $\begin{array}{l}\text { Práctica } \\
\text { Pre } \\
\text { profesio } \\
\text { nal IV }\end{array}$ & $\begin{array}{l}\text { Práctica } \\
\text { Pre } \\
\text { profesio } \\
\text { nal V }\end{array}$ & $\begin{array}{l}\text { Práctica } \\
\text { Pre } \\
\text { profesio } \\
\text { nal VI }\end{array}$ \\
\hline créditos & 1 & 1 & 1 & 1 & 1 & 1 & 1 & 3 & 14 & 11 \\
\hline $\begin{array}{l}\text { Hora } \\
\text { semanal }\end{array}$ & 2 & 2 & 2 & 2 & 2 & 2 & 2 & 4 & 20 & 20 \\
\hline $\begin{array}{l}\text { Hora } \\
\text { ciclo }\end{array}$ & 36 & 36 & 36 & 36 & 36 & 36 & 36 & 72 & 360 & 360 \\
\hline
\end{tabular}

Fuente: MINISTERIO DE EDUCACIÓN (2010a) y (2011).

Por lo tanto, la Práctica con sus diversas intencionalidades y objetos de estudio, se encuentra a lo largo de toda la formación del futuro docente, lo cual indicaría su transversalidad en el currículo, en tanto los cursos de práctica actúen como articuladores entre los cursos de la didáctica y la formación especializada. Desde esta organización curricular concurrente, se afirmaría que la competencia docente se adquiere sobre el terreno, más que en contextos académicos, en los que son frecuentes los desfases entre la teoría y la realidad (Kane, citada por Avalos, 2002, p.3).

De esta manera, se entiende que la práctica integra los distintos saberes docentes-saber práctico, empírico y el saber pedagógico-, reflexionados sobre la práctica, que se nutren de la formación teórica y del conocimiento práctico (Vezub, 2016). Asimismo, la organización dada a las prácticas en el DCBN, se ajusta a las tendencias en América Latina, que apuestan por prácticas progresivas, que incluyen actividades de campo y dispositivos pedagógicos (diarios, portafolios, ateneos, talleres de sistematización, entre otros) que propicien la reflexión sobre la práctica y reflexiones decisorias en la práctica misma.

En cuanto al número de horas de práctica, se observa en tabla 1 que, desde que ingresa del estudiante hasta el décimo ciclo, alcanza unas 1032 horas de práctica y en los dos últimos ciclos de prácticas intensivas, se concentran 720 horas, es decir, el $70 \%$ de la práctica se da en el último año de carrera. Esto es un avance frente al plan de estudios anterior al 2010, que solo reconocía un espacio de práctica pre profesional intensiva en el 
La relación entre el centro de formación y la institución educativa varía según su gestión. Cuando se trata de II.EE. de gestión pública, se concreta a través de un Convenio Interinstitucional entre las partes, que luego se presenta a las dependencias del Ministerio de Educación para su registro y, posteriormente, se envían los informes anuales sobre los convenios ejecutados, señalando logros y dificultades encontradas, así como acciones realizadas para su solución.

Para las prácticas pre profesionales con instituciones educativas de gestión privada, existe una normativa dada por el Ministerio de Trabajo y Promoción del Empleo, del año 2005, Ley $N^{\circ} 28518$. Ley sobre Modalidades Formativas Laborales y su respectiva reglamentación, que favorece una adecuada experiencia práctica en una empresa ${ }^{2}$, debidamente programada, asegurando la calidad y pertinencia de esta experiencia a través de un Convenio de Prácticas y un Plan de aprendizaje supervisado. El Ministerio de Trabajo vela por el cumplimiento de las responsabilidades registradas en el convenio y aplica las sanciones que implique su incumplimiento.

Los beneficios para los estudiantes que realizan sus prácticas pre-profesionales intensivas en la gestión privada se concretan en: recibir una subvención económica mensual equivalente al sueldo mínimo vital, como unos 270 dólares; contar con un seguro de enfermedad y accidentes; ejercer su práctica hasta 6 horas diarias y un máximo de 30 horas semanales y recibir un certificado que acredite la realización de su práctica.

En setiembre del 2018, se regulan las modalidades formativas de servicios en el Sector Público, a través del Decreto Legislativo $\mathrm{N}^{\circ} 140$. Su intención es generar mayores oportunidades e incentivos para que a futuro los jóvenes, puedan ingresar como servidores públicos. Además, se otorgan los mismos beneficios que los practicantes del régimen privado. Está pendiente la reglamentación, pero ha creado amplia expectativa en los jóvenes futuros docentes que ven esta subvención como un apoyo a su economía. Como se indicó, el documento que concretiza los acuerdos de las partes, así como obligaciones, vigencia y resolución de la Práctica Pre-profesional es el Convenio Interinstitucional entre el IESP público o privado y la Institución Educativa pública; mientras que los IESP privados que mantengan vínculo con una escuela privada, lo ejecutan en base a un Convenio de Prácticas Pre profesionales ante el Ministerio de Trabajo. En ambos, este convenio garantiza el logro de los objetivos y finalidad de las acciones de las prácticas pre-profesionales intensivas.

En cuanto a los criterios para la selección de instituciones educativas (II.EE.) señaladas en la normativa, se consideran los siguientes: la ubicación de la I.E, sus características que la hacen compatible para las prácticas pre profesionales, preferentemente públicas, disposición de sus directivos y maestros para asumir sus funciones, la identidad y el clima organizacional de la I.E., disposición de la comunidad educativa para acoger iniciativas innovadoras de los practicantes o de la institución formadora (MINISTERIO DE EDUCACIÓN, 2011, p.70-71).

Como otros criterios de selección de centros de práctica, el MINEDU ha propuesto focalizar las instituciones educativas con bajos logros de aprendizajes; también enviar a los practicantes a escuelas con buenas prácticas o con docentes innovadores y destacados en su labor profesional. Estos últimos criterios nos remiten a repensar cuáles experiencias 
serán significativas y de calidad para la formación de los practicantes, quienes enfrentan de por si contextos complejos y realidades diversas en las aulas.

Desde otro nivel de análisis, las experiencias de otros países como Argentina y Chile, que establecen una red de colaboración formal entre la escuela y las instituciones formadoras y reconocen a los docentes de aula como coformadores; en el caso de Perú, no se ha avanzado lo suficiente en trabajar la relación de las escuelas con los IESP y de institucionalizar la actividad del maestro de aula como co-formador.

Se aplica un modelo de consonancia ${ }^{3}$ en tanto, la actividad central de la práctica corresponde a la Facilitación del aprendizaje. La escuela brinda las oportunidades para que el practicante ejerza las funciones propias de un buen docente. En este modelo, los docentes formadores son responsables de: asesorar la programación, realizar acciones demostrativas, observar, asesorar, monitorear y evaluar el desempeño de los practicantes; mientras que los maestros de aula, se le solicita un rol de disposición para facilitar documentación, coordinar la ejecución de actividades y/o sesiones aprendizajes, orientar la intervención pedagógica de los practicantes en la facilitación del aprendizaje y en la gestión, dar facilidades al docente formador de la práctica, evaluar el desempeño del estudiantes practicantes en las actividades realizadas e informar sobre el desarrollo de la práctica al director de la escuela y al formador de la práctica.

En síntesis, desde la complejidad organizacional, el plan de estudios asegura las condiciones académicas para que los futuros docentes fortalezcan progresivamente sus competencias docentes, con una mayor participación en la realidad educativa. Asimismo, se regula la relación entre la escuela y el centro de formación a través de Convenios Interinstitucionales específicos, aunque la relación que se establece se tipifica como un modelo de consonancia. Por la importancia del sistema de prácticas, las instituciones formadoras empiezan a considerar muy necesario mejorar el vínculo con las escuelas que reciben los estudiantes practicantes, en los diversos tramos de su formación, con especial énfasis en las prácticas pre profesionales (Aguerrondo, 2004).

\section{DE LO NORMATIVO A LA REALIDAD DE LA PRÁCTICA PRE-PRFESIONAL}

A pesar de los marcos conceptuales, curriculares y la normativa establecida, así como los esfuerzos de las instituciones formadoras por afirmar el modelo de la Práctica con enfoque de práctica reflexiva y como un espacio de aprendizaje, donde se articule la práctica y la investigación, diversos estudios e informes respecto a cómo se conduce la práctica pre-profesional en los IESP no son alentadores.

Ugarte (2011) concluye que a pesar de los cambios introducidos en el currículo de formación docente inicial, "se han centrado básicamente en introducir la práctica preprofesional en el proceso formativo, como un curso más, aunque de naturaleza diferente" (p.27), más adelante señala que se debe pensar a fondo los procesos de articulación pues "no basta con asignar más o menos horas a la práctica o empezarla unos ciclos antes o después. Este problema se resuelve desde el enfoque de los cursos" (p.27). Ello indica que se debe revisar cómo se genera la articulación de los cursos de práctica con los otros cursos de formación general y especializada, si en los espacios de la práctica en los centros de formación, participan otros docentes de las diversas áreas o solo está el docente formador, cómo se asegura la problematización e indagación del conocimiento práctico, disciplinar y didáctico. 
Un estudio realizado en el 2016 por Pogré, Pozú y Cuenca (Cuenca, 2018), identifica respecto a las prácticas pre-profesionales que comienza al promediar la formación especializada (quinto semestre) y se intensifican en el último año de carrera, pero que son prácticas con una débil vinculación con la investigación y poco diversificadas, en cuanto a espacios y modalidades. Ello explica la afirmación de Díaz y Ñopo (2016) cuando constatan que "los futuros maestros se los prepara para la docencia en zonas urbanas, en escuelas polidocentes, sin tomar en consideración que muchos de ellos ejercerán la docencia en zonas rurales, en escuelas unidocentes o multigrado" ( $p$. 359-360).

Asimismo, el estudio de Ucelli y Ames (2008, p.164) revela que las prácticas de los estudiantes se centran más en la preocupación por las actividades y los materiales que deben emplear, que por ofrecer un nivel adecuado de los contenidos y un análisis de la secuencia de las actividades desarrolladas para que apunten al logro de las capacidades de los estudiantes. De otro lado, los espacios para la reflexión desde el curso de Práctica pre-profesional, como son los Talleres de sistematización, se realizan sin una real comprensión de lo que implica la reflexión sobre la práctica y el empleo de los dispositivos que la favorece. Como señala Revilla (2010), los docentes formadores muestran dificultades para reconocer las estrategias didácticas que promueven la práctica reflexiva "por inadecuado manejo conceptual...(aunque) todos valoran la necesidad de la reflexión en los cursos de práctica preprofesional y por ello sugieren mayor tiempo para el curso, que todos se pongan de acuerdo en cómo trabajar este aspecto" (p.16).

Estos hallazgos son preocupantes, por cuanto se estaría favoreciendo un modelo de práctica en el cual el estudiante procura imitar comportamientos o espera sea "adiestrado" por el docente formador y no se logra cimentar una práctica reflexiva sobre lo que acontece en el aula, sobre las creencias, decisiones y factores que posibilitan o limitan la actuación de los futuros docentes. Como bien señalan Louzano y Moriconi (2014, p. 36) "El problema con esos modelos de programas es que el énfasis estaría en la forma y no en el contenido ni en la reflexión sobre esas prácticas".

En un estudio de las realidades y tendencias de la formación docente inicial en el país, alerta que

La práctica profesional en la mayoría de los casos es un formalismo. Carencia de una clara estrategia que refuerce el trabajo articulado entre los centros de formación y los centros de Educación Básica. Por el contrario, es muy débil la relación entre ambas instituciones, desperdiciándose así la posibilidad de hacer de los institutos y facultades motores de la innovación e investigación en contextos muy concretos de actuación (DÍAZ, 2015, p.28).

Ello obliga a la revisión del vínculo, las relaciones de reciprocidad, la participación y compromiso entre la escuela y las instituciones formadoras, acuñando una nueva dinámica de relación. De esta manera, se buscaría que las escuelas perciban su participación en la formación de futuros docentes no como "una carga o un favor a la institución formadora" sino como una oportunidad para contribuir en la formación 
VILLAVICENCIO, L. M.

profesional de futuros docentes y mejorar las prácticas de enseñanza que se realizan en dichas escuelas (OREALC-UNESCO, 2013, p.52).

Es evidente la distancia que se establece entre lo prescriptivo o normado, como modelo del sistema de prácticas, y la práctica real en la cual se sitúan y vivencian los futuros docentes. Existe un gran desfase y serias limitaciones que nos comprometen a "mirar a fondo", a proponer y generar los cambios necesarios. Indudablemente, también existen aquellas instituciones formadoras que logran con mayor éxito la formación de competencias profesionales docentes en sus estudiantes. Sin embargo, se hace necesario acortar la distancia para garantizar calidad de la experiencia de la práctica de enseñanza $y$, en especial de las prácticas pre-profesionales.

Desafíos PARA La PRÁCtICA PRE PROFESIONAL EN LA NUEVA PROPUESTA DE FORMACIÓN DOCENTE INICIAL

En el marco del cambio curricular en el que se encuentra la formación docente inicial, se abre una oportunidad para repensar cómo se enraíza y se transversaliza un enfoque de práctica reflexiva, en tramos formativos, de mayor intensidad y complejidad durante la carrera, que atienda a la formación de un docente crítico, que construye su saber profesional en la revisión de teoría y práctica, así como en la interacción con sus pares y otros formadores; que le sirva para fundamentar sus decisiones de actuación y desarrolle sus competencias docentes en situaciones reales.

De otro lado, los hallazgos antes señalados sobre la realidad de la práctica, nos motivan a una revisión del estatuto epistemológico y organizacional, para proponer nuevos modos de concretar las prácticas pre-profesionales que nos permitan superar algunos de los desafíos identificados en nuestro país.

- Asegurar una experiencia de calidad de las prácticas pre-profesionales

Este es el principal desafío, por cuanto implicará generar mayor reflexión y acuerdos entre las instituciones formadoras, tanto del ámbito público como privado, sobre lo que es asegurar una "experiencia de calidad" en las prácticas pre-profesionales de los futuros docentes. Se espera que las prácticas permitan "aprender a enseñar" ofreciendo oportunidades para "practicar" la docencia, con diversos grupos y realidades complejas, reflexionando críticamente sobre su práctica, su rol como enseñante y la docencia como profesionalidad (Kane, 2002). Ello es contrario a una experiencia de formación que conduce a "reproducir hábitos tradicionales y modelos acríticos de la profesión docente" (Rodríguez y Grilli, 2016, p.202) porque se descuidó el seguimiento y la propuesta de dispositivos que favorezcan una experiencia distinta.

La forma cómo se desarrollen las prácticas pre-profesionales deben ser de conocimiento de todos los agentes que intervienen, definiéndose adecuados estándares, contenidos, metodologías para una práctica reflexiva e instrumentos de seguimiento y de evaluación para uso de los distintos agentes. En ese sentido, la planificación, funcionamiento y evaluación de las prácticas, así como la toma de decisiones, tendría que ser compartida y dialogada con los agentes que participan en la escuela y en los centros de formación. 
- Construir una didáctica de la práctica de la enseñanza

Sobre la práctica, Kane (2002) señala que no solo se trata de que los futuros docentes tengan oportunidades de enseñar y ejercer las funciones del docente, sino que "necesitan comprender los imperativos pedagógicos, sociales, culturales y educativos que rigen la práctica docente, o sea, examinar y reconciliar la teoría y la realidad de la enseñanza" $(2002$, p.3). Asimismo, resulta importante reconocer las expectativas de los estudiantes y los modelos de hacer docencia que traen (habitus profesional) para que los revisen, critiquen, comprendan y construyan un sentido y finalidad de la práctica, que se aproxime más al modelo de reflexión desde y sobre la práctica.

Se coincide con Aguerrondo (2004) en que se debe hacer explícito en el nuevo currículo de formación docente, una didáctica de la práctica de enseñanza, en tanto la práctica docente se enseña, así como se enseña a reflexionar, a mirar de modo crítico la acción docente y ello implica formar en esta línea a los docentes que acompañan la práctica y a los docentes de las escuelas.

- Una renovada relación basada en la colaboración entre los centros de formación y la escuela

Se trata de superar el desencuentro entre los centros de formación y las escuelas que reciben a los docentes en formación. Para ello, será necesario generar un acuerdo sobre un nuevo tipo de relación que se ajuste al enfoque de formación y que sea aceptado por todos los que participan en la práctica.

En el estudio realizado por Vaillant (2018) destaca que los centros de formación requieren de escuelas que sean "contextos de práctica, de aplicación, de vida cotidiana y, en definitiva, de socialización" para que completen el marco general formativo de sus estudiantes (2018, p.20) y, a su vez, las escuelas requieren ensayar nuevas formas de aprender y estrategias para enseñar, que podría potenciarse con el intercambio y apoyo del equipo de docentes de la institución formadora.

Es así que el modelo de resonancia colaborativa parece una alternativa. Este supone una responsabilidad compartida en la formación y es una vía importante para la innovación pedagógica. Su implementación requerirá la revisión de los criterios para la selección de los centros de práctica, en tanto se tendría que añadir el compromiso y la disposición de la escuela y de sus profesores, para convertirse en una "escuela que aprende" y por parte de la institución formadora, el cambio de una relación vertical que detenta el poder del conocimiento a una relación horizontal, de conocimiento compartido y construcción de proyectos de innovación en base a intereses comunes.

De otro lado, también será importante asegurar que el Convenio de Prácticas Interinstitucionales exprese un marco común sobre la formación y la profesionalidad docente, entendida como una acción colaboradora y de nuevos vínculos que beneficien tanto a la institución escolar como a la institución formadora.

- Reconocimiento de estatus a los docentes de aula colaboradores 
VILLAVICENCIO, L. M.

Se reconoce que la formación inicial de los estudiantes no solo depende de los docentes que tienen a su cargo los cursos de práctica en el centro de formación, sino que juegan un rol muy importante el director(a) y los docentes, que aceptan a los estudiantes de práctica en la institución educativa y en las aulas.

Estos docentes modelan e inciden en la construcción de la identidad profesional docente de los estudiantes de práctica, se constituyen en "modelos" de profesionalidad y de un "estilo" de hacer enseñanza, por lo que se les suele denominar: coformadores o docentes colaboradores. No se trata de depender de la "buena voluntad" del docente colaborador, sino que se reconozca su participación en la formación de los practicantes y que ello implica dedicación, tiempo, preparación que se añade a las labores que debe atender.

En la actual Ley de Reforma Magisterial $N^{\circ} 29944$, se indica cuatro áreas de desempeño laboral, para el ejercicio de cargos y funciones de los profesores para la Carrera Pública Magisterial. Una de las áreas se denomina Formación docente, que comprende a los profesores que "realizan funciones de acompañamiento pedagógico, de mentoría a profesores nuevos, de coordinador y/o especialista en programas de capacitación, actualización y especialización de profesores al servicio del Estado" (CONGRESO DE LA REPÚBLICA DEL PERÚ, 2016, p.27) para que mejoren su práctica docente. Esta función puede ser ampliada no solo a sus pares, sino a los futuros docentes o practicantes, de esta forma será reconocida su labor en el escalafón magisterial.

- La reflexión sobre la práctica como un continum en el desarrollo profesional docente

El desarrollo de la profesionalidad y la identidad docente es uno de los dominios expresados en el Marco del Buen Desempeño Docente. Este se traduce en la competencia vinculada con la reflexión sobre la práctica y la experiencia institucional en orden a que el docente construya y afirme su identidad y responsabilidad profesional. Sin embargo, asumirse como un docente reflexivo no es sencillo, más aun cuando ha sido formado bajo otro modelo tradicional o técnico.

Se considera un desafío para la formación inicial, que en las instituciones formadoras y los docentes formadores, enseñen a los futuros docentes a mirar la práctica desde una actitud y postura reflexiva, que les permita reconceptualizarla aplicando una didáctica de la práctica de enseñanza durante su formación. Es decir, se requiere "encarnar" el enfoque reflexivo práctico, para que no sea solo un discurso que repite lo que está escrito en los documentos que alcanza el Ministerio de Educación. Es urgente una profunda comprensión y manejo adecuado de una formación docente desde un enfoque reflexivo y práctico. Ello se debe traducir en las experiencias formativas en los centros de formación y, por ende, en la calidad de las experiencias de la práctica y práctica pre-profesional.

Los futuros docentes deben encontrar en sus prácticas una conexión entre lo que ven en las escuelas y lo que aprenden en las clases; asimismo, múltiples posibilidades para utilizar de forma regular actividades y dispositivos de reflexión y análisis de experiencias, que viven los practicantes y los coformadores, para la mejora de la formación de esos maestros y de las prácticas docentes en la escuela. De esta manera, cuando ingresen a la 
carrera magisterial, la reflexión en y sobre su práctica sea intrínseca a su desarrollo profesional.

Artículo recibido en: 24/02/2019

Aprobado para publicación en: 27/06/2019

\section{PRÁTICA PRÉ-PROFISSIONAL NA FORMAÇÃO INICIAL DE ENSINO DAS POLÍTICAS DE ENSINO NO PERU}

RESUMO: Nesta década, a formação de professores no Peru passa por mudanças marcadas pelas políticas dos professores, comprometidas com o exercício de um ensino baseado em padrões vinculados à Carreira Pública de Ensino. Este artigo fornece uma visão geral das políticas que regulam a formação de futuros professores para focalizar seu foco na prática pré-profissional de professores treinados nos Institutos de Educação Pedagógica Superior. As práticas intensivas são analisadas a partir de sua complexidade epistemológica e organizacional, como um espaço de aprendizado, um processo complexo e único, além de uma área do currículo que desenvolve habilidades profissionais. Termina com desafios que permitem o diálogo entre os agentes responsáveis pela formação de professores em nosso país.

PALAVRAS - CHAVE: Prática pré-profissional. Formação inicial de professores. Políticas de ensino.

\section{PRE-PROFESSIONAL PRACTICE IN INITIAL TEACHING TRAINING FROM TEACHING POLICIES IN PERU}

ABSTRACT: In this decade, teacher training in Peru is undergoing changes marked by teacher policies, which are committed to the exercise of a teaching based on standards linked to the Teaching Public Career. This article provides an overview of the policies that regulate the training of future teachers to focus their focus on the pre-professional practice of teachers who are trained in the Institutes of Higher Pedagogical Education. Intensive practices are analyzed from their epistemological and organizational complexity, as a learning space, a complex and unique process, as well as an area of the curriculum that develops professional skills. It ends with challenges that allow the dialogue between the agents responsible for teacher training in our country.

KEYWORDS: Pre-professional practice. Initial teacher training. Teaching policies.

\section{NOTAS}

1) El modelo curricular consecutivo asegura el domino disciplinar, certificado con algún grado académico, luego se continúa por dos o tres años con formación especializada en pedagogía. En este modelo curricular, las prácticas de enseñanza se concentran durante la formación pedagógica de forma intensiva. 
VILLAVICENCIO, L. M.

2) La institución educativa de gestión privada entra en la categoría de empresa y es afectada por la mencionada Ley.

3) En la actualidad, se cuenta con evidencias de cómo funcionan distintos modelos de colaboración entre instituciones formadoras y las escuelas (Escobar, 2007, Rodríguez y Grilli, 2016), en base a la propuesta de Marcelo y Estebaranz (1998) quienes plantearon los siguientes modelos: de yuxtaposición, de consonancia en torno a un perfil del buen profesor, de disonancia y, de resonancia colaborativa.

\section{REFERENCIAS}

AGUERRONDO, Inés. Los desafíos de la política educativa relativos a las reformas de la formación docente. En PREAL. Maestros en América Latina: Nuevas Perspectivas sobre su Formación y Desempeño. 1.ed. Santiago: San Marino, 2004, p. 97- 141.

AVALOS, Beatrice. Formación docente: reflexiones, debates, desafíos e innovaciones Perspectivas. Vol. XXXII, $\mathrm{n}^{\circ} 3$, sept. 2002, p. 1-9. Disponible en:

$<$ http://www.ibe.unesco.org/fileadmin/user_upload/archive/Publications/Prospects/Pro spectsPdf/123s/123s.pdf>. Acceso en: 5/1/2019.

CONGRESO DE LA REPÚBLICA DEL PERÚ. Ley N³0512, Ley de Institutos y Escuelas de Educación Superior y de la Carrera Pública de sus docentes. Diario Oficial El Peruano №1621490-1, Lima, 2 de noviembre de 2016.

. Ley № 28044, Ley General de Educación. Diario Oficial El Peruano № 8437, Lima, 28 julio 2003.

. Ley № 29944, Ley de Reforma Magisterial. Diario Oficial El Peruano № 479340, Lima, 23 de noviembre del año 2012. julio de 2014.

. Ley $N^{\circ} 30220$, Ley Universitaria. Diario Oficial El Peruano N 527211, Lima, 9 de

. Ley $\mathrm{N}^{\circ} 28518$, Ley sobre modalidades formativas laborales. Diario Oficial El Peruano $N^{\circ}$ 527211 Lima, 20 de mayo de 2005.

. Decreto Supremo N ${ }^{\circ}$ 007-2005-TR. Reglamento de la Ley sobre modalidades formativas laborales. Diario Oficial El Peruano, Lima, 19 de setiembre de 2005.

Decreto Supremo N ${ }^{\circ} 010-2017-M I N E D U$, Ley de Institutos y Escuelas de Educación Superior y de la Carrera Pública de sus docentes. Diario Oficial El Peruano, Lima, 25 de agosto de 2017.

CUENCA, Ricardo. La formación inicial docente: el Perú en clave latinoamericana. En: II SEMINARIO NACIONAL DE LA RED KIPUS PERÚ. POLITICAS EDUCATIVAS DOCENTES DESDE LA FORMACIÓN INICIAL. Lima, 2018. Disponible en: 
$<$ http://www.redkipusperu.org/inicio/wp-content/uploads/2018/09/ConferenciaRicardo-Cuenca-24-8-18.pdf. >. Diapositivas de PowerPoint. Acceso en: 29/12/2018

DIAZ, Hugo. Formación docente en el Perú. Realidades y tendencias. Lima: GRAMBS, 2015.

DÍAZ, Juan José; ÑOPO, Hugo. La carrera docente en el Perú. En: GRADE. Investigación para el desarrollo en el Perú: once balances. Lima: GRADE, 2016. p. 353- 401. Disponible en:

<http://biblioteca.clacso.edu.ar/Peru/grade/20170417125048/carreradocente_JJDHN_3 5.pdf>. Acceso en: 6/01/2019.

DIFOID. Censo a Institutos y Escuelas de Educación Superior pedagógica 2015. Algunos datos relevantes. En: REUNIÓN DE PRESENTACIÓN DE LINEAMIENTOS Y POLÍTICAS A SEGUIR EN EL MARCO DE LA REFORMA DE LA FORMACIÓN INICIAL DOCENTE. Recuperado de: $<$ http://www.minedu.gob.pe/superiorpedagogica/reunion-depresentacion-de-lineamientos-y-politicas-a-seguir-en-el-marco-de-la-reforma-de-laformacion-inicial-docente/> Lima, MINEDU, 2017a.

. Hacia la Excelencia de los Institutos de Educación Superior Pedagógicos. En: SEMINARIO NACIONAL POLÍTICAS DE DESARROLLO DOCENTE AL 2021 [diapositivas de PowerPoint]. Recuperado de <http://www.redkipusperu.org/inicio/wpcontent/uploads/2017/07/Kipus-Jessica-Soto-DIFOID-2017-06-03.pdf > Lima, Red Kipus Perú, 2017b.

ESCOBAR, Nancy. La práctica profesional docente desde la perspectiva de los estudiantes practicantes y tutores. Acción Pedagógica, № 16, p .182-193, 2007. Disponible en:<https://dialnet.unirioja.es/descarga/articulo/2968746.pdf >. Acceso en: 8/01/2019.

KANE, Ruth. Cómo enseñamos a los docentes: nuevos modos de teorizar sobre la práctica y poner en práctica la teoría. Perspectivas. Vol. XXXII, n³, sept. 2002, p. 1-22. Disponible en:

$<$ http://www.ibe.unesco.org/fileadmin/user_upload/archive/Publications/Prospects/Pro spectsPdf/123s/kanes.pdf.>. Acceso en: 5/1/2019.

LOUZANO, Paula; MORICONI, Gabriela. Visión de la docencia y características de los sistemas de formación docente. En: OREALC-UNESCO. Temas críticos para formular nuevas políticas docentes en América Latina y El Caribe: el debate actual. UNESCO, 2014 Chile, p.10-52.

MARCELO, Carlos; ESTEBARANZ, Araceli. Modelos de colaboración entre la universidad y las escuelas en la formación del profesorado. Revista de Educación, n. 317, p. 97-120, 
VILLAVICENCIO, L. M.

1998. Disponible en:

$<$ https://idus.us.es/xmlui/bitstream/handle/11441/31841/Modelos_de_colaboracion_en tre_la_universidad.pdf;sequence $=1>$. Acceso en: 12/12/2018

MINISTERIO DE EDUCACIÓN. Diseño Curricular Básico Nacional para la carrera Profesional de Profesor de Educación Primaria. Lima, 2010a. 144 p.

Resolución Directoral 0651-2010-ED. Lineamientos Nacionales para el Desarrollo de la Práctica Pre-profesional en carreras docentes en Institutos y Escuelas de Educación Superior públicos y privados. Lima, 19 de agosto de 2010.

Guía de Práctica para Institutos y Escuelas de Educación Superior con Carreras Pedagógicas. Lima, 2011. 124 p.

OREALC-UNESCO. Antecedentes y Criterios para la Elaboración de Políticas Docentes en América Latina y el Caribe. Chile: UNESCO, 2013, 170 p.

REVILLA, Diana. La práctica reflexiva durante el desarrollo de la práctica pre - profesional docente. Ponencia presentada en Congreso Iberoamericano de Educación- Metas 2021. 13 al 15 de setiembre. Buenos Aires: Ministerio de Educación de Argentina, SEGIB, OEI y ACDI, 2010b, p. 1-18. Disponible en $<$ www.adeepra.org.ar/congresos/.../DOCENTES/RLE2144_Revilla.pdf>.Acceso en: 15/12/2018.

RODRIGUEZ, Eduardo; GRILLI, Javier. Desafíos del modelo de práctica resonancia colaborativa en la formación inicial de docentes. Educar em Revista, Curitiba, n.62, p.199227, out./dez. 2016. Disponible en: <http://www.scielo.br/pdf/er/n62/1984-0411-er-6200199.pdf >. Acceso en: 5/01/2019.

UCELLI, Francesca; AMES, Patricia. Formando futuros maestros: observando las aulas de institutos superiores pedagógicos públicos En: GRADE. Análisis de programas, procesos y resultados educativos en el Perú: contribuciones empíricas para el debate. Lima: GRADE, 2008. p. 131- 175. Disponible en: <http://bibliotecavirtual.clacso.org.ar/Peru/grade/20100406102729/analisis-4.pdf>. Acceso en: 21/ 12/2018

UGARTE, Darío. Políticas de formación y desarrollo docente: Balance y temas críticos. Lima: USAID/PERU/SUMA, 2011, 35 p. Disponible en: <http://mapeal.cippec.org/wpcontent/uploads/2014/05/Pol\%C3\%ADticas-de-formaci\%C3\%B3n-y-desarrollodocentes-balances-y-temas-cr\%C3\%ADticos.pdf>. Acceso en: 4/01/2019

VAILLANT, Denise. Estudios exploratorios sobre modelos organizaciones y pedagógicos de instituciones dedicadas a la formación docente inicial. Un análisis en clave comparada. Disponible en: < http://panorama.oei.org.ar/ dev2/wp- 
content/uploads/2018/05/Informe-final-Estudio-formaci\%C3\%B3n-docente-INFOD.pdf > Buenos Aires, UNESCO-IIEP, 2018.

VELASCO, Liriama. Políticas de formación inicial docente. En: II SEMINARIO NACIONAL DE LA RED KIPUS PERÚ. POLÍTICAS EDUCATIVAS DOCENTES DESDE LA FORMACIÓN INICIAL. Lima, 2018. Disponible en: http://www.redkipusperu.org/inicio/wpcontent/uploads/2018/09/Conferencia-LIRIAMA-VELAZCO-Fomaci\%C3\%B3n-Inicial.pdf. Diapositivas de PowerPoint.

VEZUB, Lea. F. Los saberes en la formación inicial. La perspectiva de los formadores. Pensamiento Educativo, Santiago, 53(1), 1-14.2016. Disponible en: <https://goo.gl/V1gBwq>. Acceso en: 27/12/2018

Lileya Manrique VillaVicencio: Mestre Magíster en Educación con mención en Currículo (Pontificia Universidad Católica del Perú). Licenciado en Educación con especialidad en Educación Primaria (Pontificia Universidad Católica del Perú). Docente ordinário - principal. Docente a tiempo completo (DTC). Departamento Académico de Educación - Sección Educación.

Orcid: https://orcid.org/0000-0002-1044-6298

E-mail: Imanriq@pucp.edu.pe

Este periódico utiliza a licença Creative Commons Attribution 3.0, para periódicos de acesso aberto (Open Archives Iniciative - OAI). 